\title{
A Study of Quality in Primary Education Combined Disjoint Block Neutrosophic Cognitive Maps (CDBNCM)
}

\author{
A.Victor Devadoss ${ }^{1}$, M. Clement Joe Anand ${ }^{2}$, A. Joseph Bellarmin ${ }^{3}$ \\ ${ }^{1}$ Head \& Associate Professor, Department of Mathematics, Loyola College, Chennai - 34,India. \\ ${ }^{2}$ Ph.D Research Scholar, Department of Mathematics, Loyola College, Chennai-34, India. \\ ${ }^{3}$ M.Phil Mathematics, Department of Mathematics, Loyola College, Chennai-34, India.
}

\begin{abstract}
Quality in primary education has been classified into five factors involving learners, content, processes, environment and outcomes. In this paper we analyzed, quality in primary education in Chennai and find out its solution using Neutrosophic cognitive maps (NCMS), which is the generalization of fuzzy cognitive maps (FCMS) defined by W.B. Vasantha Kandasamy and Florentine Smarandache. This paper has five sections. First section gives information about development of fuzzy cognitive maps and Neutrosophic cognitive maps. Second section gives the preliminaries of FCMS and NCMS. In section three, we give the description of the problem; final section gives the conclusion based on our study.
\end{abstract}

\section{Introduction:}

IN 1965, L.A. Zadeh has introduced a mathematical model called Fuzzy cognitive Maps. After a decade in the year 1976, Political scientist R. axelord[7] used this fuzzy model to study decision making in social and political systems. Then B.Kosko [1], [2], [3], enhanced the power of cognitive maps considering fuzzy vales for the concepts of the cognitive map and fuzzy degrees of interrelationships between concepts. FCMs can successfully represent knowledge and human experience, introduced concepts to represent the essential elements and the cause and effect relationships among the convenient simple and powerful tool, Which is used in numerous fields such as social economical and medical etc. In this paper we use the Neutrosophic cognitive maps (NCMs) created by and Florentine Smarandache [8],[9], which is an extension/ combination of the fuzzy cognitive maps (FCMS) in which inderminacy is included. It has also become very essential that the notation of Neutrosophic logic plays a vital role in several of the real world problems like law, medicine, industry, finance, IT, stocks and share etc. Here we defining about education.

International Journal of Business Intelligents (IJBI) www.ijbui.com

\section{Quality education includes:}

- Learners who are healthy, well-nourished and ready to participate and learn, and supported in learning by their families and communities;

- Environments that are healthy, safe, protective and gender-sensitive, and provide adequate resources and facilities;

- Content that is reflected is relevant curricula and materials for the acquisition of basic skills, especially in the areas of literacy, numeracy and skills for life, and knowledge in such areas as gender, health, nutrition, HIV/AIDS prevention and peace.

- Processes through which trained teachers use child-centered teaching approaches in well-managed classrooms and schools and skillful assessment to facilitate learning and reduce disparities.

- Outcomes that encompass knowledge, skills and attitudes, and are linked to national goals for education and positive participation in society

This definition allows for an understanding of education as a complex system embedded in a political, cultural and economic context. It is important to keep in mind education's systemic nature, however; these dimensions 
are interdependent, influencing each other in many ways.

\section{Preliminaries}

Fuzzy Cognitive Maps (FCMs) are more applicable when the data in the first place is an unsupervised one. The FCMs work on the opinion of experts. FCMs model the world as a collection of classes and causal relations between classes.

\subsection{Definition}

A NCMs is a directed graph with concepts like policies, events etc, as nodes and causalities as edges. It represents causal relationship between concepts.

\subsection{Definition}

When the nodes of the NCM are fuzzy sets then they are called as fuzzy nodes.

\subsection{Definition}

NCMs with edge weights or causalities from the set $\{-1,0,1, I\}$ are called simple NCMs.

\subsection{Definition}

Let $C_{i}$ and $C_{j}$ denote the two nodes of the NCM. The directed edge from $C_{i}$ to $C_{j}$ denotes the causality of $\mathrm{C}_{i}$ on $\mathrm{C}_{\mathrm{j}}$ called connections. Every edge in the NCM is weighted with a number in the set $\{-1,0,1$, I\}. Let $\mathrm{e}_{\mathrm{ij}}$ be the weight of the directed edge $\mathrm{C}_{\mathrm{i}} \mathrm{C}_{\mathrm{j}}, \mathrm{e}_{\mathrm{ij}} \in\{-1,0,1, I\} . \mathrm{e}_{\mathrm{ij}}=0$ if $\mathrm{C}_{\mathrm{i}}$ does not have any effect on $\mathrm{C}_{\mathrm{j}}, \mathrm{e}_{\mathrm{ij}}=1$ if increase (or decrease) in $\mathrm{C}_{\mathrm{i}}$ causes increase (or decreases) in $C_{j} . e_{i j}=-1$ if increase (or decrease) in $C_{i}$ causes decrease (or increase) in $C_{j} \cdot e_{i j}=I$ if the relation or effect of $\mathrm{C}_{\mathrm{i}}$ on $\mathrm{C}_{\mathrm{j}}$ is an indeterminate.

\subsection{Definition}

Let $C_{1}, C_{2}, \ldots, C_{n}$ be nodes of a NCM. Let the neutrosophic matrix $\mathrm{N}(\mathrm{E})$ be defined as $N(E)=\left(e_{i j}\right)$ where $e_{i j}$ is the weight of the directed edge $\mathrm{CiC} \mathrm{j}$, where eij $\in\{-1,0,1, I\}$. $\mathrm{N}(\mathrm{E})$ is called the neutrosophic adjacency matrix of the NCM.

\subsection{Definition}

Let $\mathrm{C} 1, \mathrm{C} 2, \ldots, \mathrm{Cn}$ be the nodes of an NCM. $\mathrm{A}=(\mathrm{a} 1, \mathrm{a} 2, \ldots$, an $)$ where ai $\in\{0,1, I\} . \mathrm{A}$ is called the instantaneous state neutro-sophic vector and it denotes the on-off-indeterminate state position of the node at an instant. ai $=0$ if ai is off (no effect) ai = 1 if ai is on (has effect) ai $=I$ if ai is indeterminate (effect cannot be deter-mined) for $\mathrm{i}=1,2, \ldots, \mathrm{n}$.

\subsection{Definition}

Let $\mathrm{C} 1, \mathrm{C} 2, \ldots, \mathrm{Cn}$ be the nodes of and FCM. Let be the edges of the NCM. Then the edges form a directed cycle. An NCM is said to be cyclic if it possesses a directed cyclic. An NCM is said to be acyclic if it does not possess any directed cycle.

\subsection{Definition}

An NCM with cycles is said to have a feedback.

\subsection{Definition}

When there is a feedback in an NCM, i.e, when the causal relations flow through a cycle in a revolutionary manner the NCM is called a dynamical system.

\subsection{Definition}

Let be a cycle. When $\mathrm{Ci}$ is switched on and if the causality flows through the edges of a cycle and if it again causes $\mathrm{Ci}$, we say that the dynamical system goes round and round. This is true for any node $\mathrm{Ci}$ for $\mathrm{i}=1,2, \ldots, \mathrm{n}$. The equilibrium state for this dynamical system is called the hidden pattern.

\subsection{Definition}

If the equilibrium state of a dynamical system is a unique state vector, then it is called a fixed point. Consider a NCM with $\mathrm{C} 1, \mathrm{C} 2, \ldots$, $\mathrm{Cn}$ as nodes. For example let us start the dynamical system by switching on $\mathrm{C} 1$. Let us assume that the NCM settles down with $\mathrm{C} 1$ and $\mathrm{Cn}$ on i.e., the state vector remain as (1, $0,0, \ldots, 1)$ this neutrosophic stage vector $(1$, $0, \ldots, 0,1)$ is called fixed point. 


\subsection{Definition}

If the NCM settles down with a neutrosophic state vector re-peating in the form $\mathrm{A} 1 \rightarrow \mathrm{A} 2 \rightarrow \ldots \rightarrow \mathrm{Ai} \rightarrow \mathrm{A} 1$ then this equilibrium is called a limit cycle of the NCM.

\subsection{Definition}

Finite number of NCMs can be combined together to produce the point effect of all the NCMs. If $\mathrm{N}(\mathrm{E} 1), \mathrm{N}(\mathrm{E} 2), \ldots, \mathrm{N}(\mathrm{Ep})$ be the neutrosophic adjacency matrices of a NCM with nodes $\mathrm{C} 1, \mathrm{C} 2, \ldots, \mathrm{Cn}$ then the combined NCM is got by adding all the neu-trosophic adjacency matrices $\mathrm{N}(\mathrm{E} 1), \mathrm{N}(\mathrm{E} 2), \ldots, \mathrm{N}(\mathrm{Ep})$. We de-note the combined NCMs adjacency neutrosophic matrix by $\mathrm{N}(\mathrm{E})=$ $\mathrm{N}(\mathrm{E} 1)+\mathrm{N}(\mathrm{E} 2)+\ldots+\mathrm{N}(\mathrm{Ep})$.

\section{Method of Determining the Hidden Pattern}

Let $\mathrm{C} 1, \mathrm{C} 2, \ldots, \mathrm{Cn}$ be the nodes of an NCM, with feedback, Let $\mathrm{E}$ be the associated adjacency matrix. Let us find the hidden pattern when $\mathrm{C} 1$ is switched on when an input is given as the vector $\mathrm{A} 1=(1,0, \ldots, 0)$, the data should pass through the neutrosophic matrix $\mathrm{N}(\mathrm{E})$. This is done by multiplying A1 by the matrix $\mathrm{N}(\mathrm{E})$. Let $\mathrm{A} 1 \mathrm{~N}(\mathrm{E})=(\mathrm{a} 1, \mathrm{a} 2, \ldots$, an) with the threshold operation that is by replacing ai by 1 if ai $>\mathrm{k}$ and ai by 0 if ai $<\mathrm{k}$ ( $\mathrm{k}-\mathrm{a}$ is a suitable positive integer) and ai by $I$ if ai is not integer. We update the resulting concept; the concept $\mathrm{C} 1$ is included in the updated vector by making the first coordinate as 1 in the resulting vector. Suppose $\mathrm{A} 1 \mathrm{~N}(\mathrm{E}) \rightarrow \mathrm{A} 2$ then consider $\mathrm{A} 2 \mathrm{~N}(\mathrm{E})$ and repeat the same procedure. This procedure is repeated till we get a limit cycle or a fixed point.

\section{Concepts of the problem:}

What does quality mean in the context of education? Many definition of quality in education exist, testifying to the complexity and multifaceted nature of the concept. The terms efficiency, effectiveness, equity and quality have often been used synonymously. The objective of the study is to assess the quality in primary education. For that, using linguistic questionnaire and the expert's opinion we have taken the following concepts.

The following concepts are taken as the main nodes of our problem.

$\mathrm{B}_{1}-$ Good health and nutrition.

$\mathrm{B}_{2}$-Early childhood psychological development experiences.

$\mathrm{B}_{3}-$ Regular attendance for learning.

$\mathrm{B}_{4}-$ Family support for learning.

$\mathrm{B}_{5}-$ Quality of school facilities.

$\mathrm{B}_{6}$ - Interaction between school infrastructure and other quality dimensions.

$\mathrm{B}_{7}$ - Class size/Class strength

$\mathrm{B}_{8}$ - Peaceful, Safe environments for children

$\mathrm{B}_{9}-$ Teachers' behaviours that affect safety

$\mathrm{B}_{10}$ - Effective school discipline polices

$\mathrm{B}_{11}$ - Inclusive environments

$\mathrm{B}_{12}-$ Non-violence

$\mathrm{B}_{13}$ - Provision of health services

$\mathrm{B}_{14-}$ Student-centred, non-discriminatory, standards-based curriculum structures

$\mathrm{B}_{15}$ - Student access to subjects taught at school

$\mathrm{B}_{16}$ - Uniqueness of local and national content

$\mathrm{B}_{17}$ - Literacy

$\mathrm{B}_{18}$ - Numeracy

$\mathrm{B}_{19}-$ Life skills

$\mathrm{B}_{20}-$ Peace education

These 20 attributes are divided into 4 classes $\mathrm{C}_{1}, \mathrm{C}_{2}, \mathrm{C}_{3}, \mathrm{C}_{4}$ with 5 in each class.

Let $C_{1}=\left\{B_{1}, B_{5}, B_{9}, B_{15}, B_{18}\right\}, C_{2}=\left\{B_{4}, B_{7}\right.$, $\left.B_{10}, B_{13}, B_{17}\right\}, C_{3}=\left\{B_{2}, B_{6}, B_{12}, B_{16}, B_{20}\right\}$ and $\mathrm{C}_{4}=\left\{\mathrm{B}_{3}, \mathrm{~B}_{8}, \mathrm{~B}_{11}, \mathrm{~B}_{14}, \mathrm{~B}_{19}\right\}$.

Now we take the expert opinion for each of these classes and take the matrix associated with the combined disjoint block NCMs.

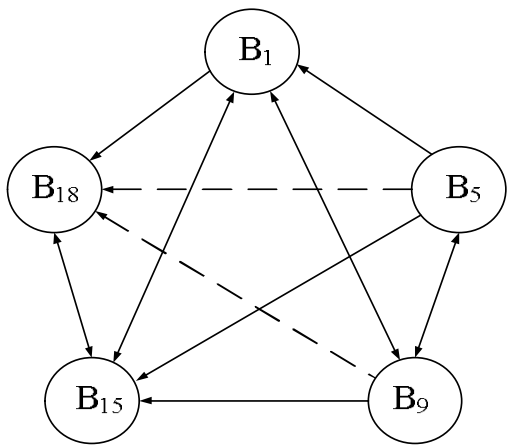


The expert's opinion for the class $C_{1}=\left\{B_{1}\right.$, $\left.\mathrm{B}_{5}, \mathrm{~B}_{9}, \mathrm{~B}_{15}, \mathrm{~B}_{18}\right\}$ in the form of the directed graph.According to this expert, the attribute good health and nutrition is interrelated to teacher's behaviours that affect safety. The attribute student access to subjects taught at school is interrelated to Numeracy. The attribute good health and nutrition is interrelated to student access to subjects taught at school.

The related connection matrix $\mathrm{M}_{1}$ is given by

$$
M_{1}=\left[\begin{array}{lllll}
0 & 0 & 1 & 1 & 1 \\
1 & 0 & 0 & 1 & I \\
1 & 0 & 0 & 1 & I \\
1 & 0 & 0 & 0 & 1 \\
0 & 0 & 0 & 1 & 0
\end{array}\right]
$$

The directed graph is given by the expert on $\mathrm{B}_{4}, \mathrm{~B} 7, \mathrm{~B}_{10}, \mathrm{~B}_{13}, \mathrm{~B}_{17}$ which forms the class $\mathrm{C}_{2}$.

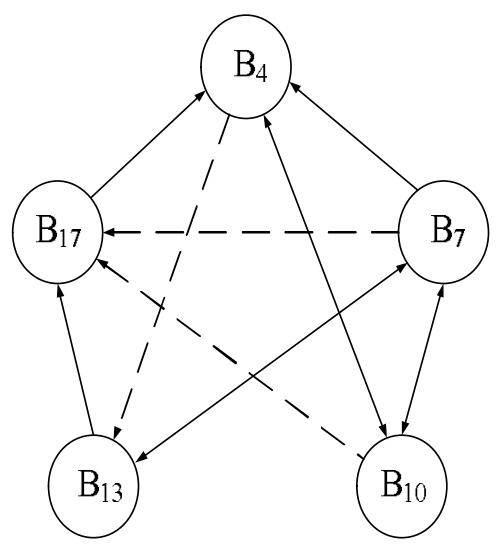

According to this expert, the attribute class size/class strength is interrelated to provision of health services. The related connection matrix $\mathrm{M}_{2}$ is given by

$$
M_{2}=\left[\begin{array}{lllll}
0 & 0 & 0 & I & 0 \\
1 & 0 & 1 & 1 & I \\
0 & 0 & 0 & 0 & I \\
0 & 1 & 0 & 0 & 1 \\
1 & 0 & 0 & 0 & 0
\end{array}\right]
$$

The expert's opinion for the class $C_{3}=\left\{B_{2}, B_{6}\right.$, $\left.\mathrm{B}_{12}, \mathrm{~B}_{16}, \mathrm{~B}_{20}\right\}$ in the form of the directed graph.

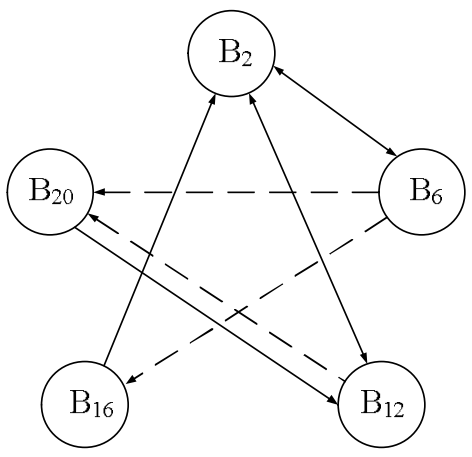

According to this expert, the attribute early childhood psychosocial development experiences is interrelated to non-violence.

The related connection matrix $\mathrm{M}_{3}$ is given by

$$
M_{3}=\left[\begin{array}{lllll}
0 & 1 & 1 & 0 & 0 \\
1 & 0 & 0 & I & 0 \\
1 & 0 & 0 & 0 & I \\
1 & 0 & 0 & 0 & 0 \\
0 & 0 & 1 & 0 & 0
\end{array}\right]
$$

The expert's opinion for the class $C_{4}=\left\{B_{3}, B_{8}\right.$, $\left.\mathrm{B}_{11}, \mathrm{~B}_{14}, \mathrm{~B}_{19}\right\}$ in the form of the directed graph.

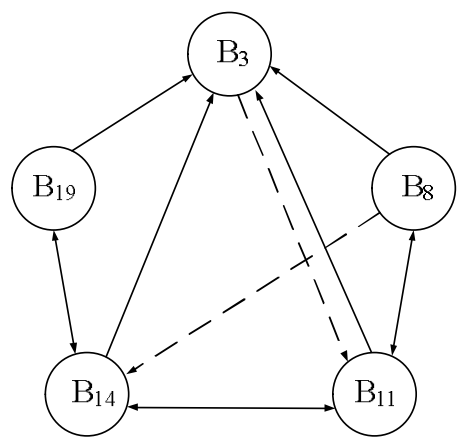

The related connection matrix is given by

$$
M_{4}=\left[\begin{array}{lllll}
0 & 0 & I & 0 & 0 \\
1 & 0 & 1 & 0 & 0 \\
1 & 1 & 0 & 1 & 0 \\
1 & I & 1 & 0 & 1 \\
1 & 0 & 0 & 1 & 0
\end{array}\right]
$$


According to this expert, the attribute peaceful, safe environments for children is interrelated to inclusive environment. The attribute inclusive environments is interrelated to student- centered, non- discriminatory, standards-based curriculum structures. The attribute student- centered, non- discriminatory, standards-based curriculum structures is interrelated to life skills.

\begin{tabular}{|c|c|c|c|c|c|c|c|c|c|c|c|c|c|c|c|c|c|c|c|}
\hline$B_{1}$ & {$[0$} & 0 & 0 & 0 & 0 & 0 & 0 & 1 & 0 & 0 & 0 & 0 & 0 & 0 & 0 & 0 & 1 & & \\
\hline$B_{2}$ & 0 & 0 & 0 & 0 & 1 & 0 & 0 & 0 & 0 & 0 & 1 & 0 & 0 & 0 & 0 & 0 & 0 & 0 & \\
\hline$B_{3}$ & 0 & 0 & 0 & 0 & 0 & 0 & 0 & 0 & 0 & $I$ & 0 & 0 & 0 & 0 & 0 & 0 & 0 & 0 & \\
\hline $3_{4}$ & 0 & 0 & 0 & 0 & 0 & 0 & 0 & 0 & 0 & 0 & 0 & $I$ & 0 & 0 & 0 & 0 & 0 & 0 & \\
\hline$\beta_{5}$ & 0 & 0 & 0 & 0 & 0 & 0 & 0 & 0 & 0 & 0 & 0 & 0 & 0 & 1 & 0 & 0 & 0 & 0 & \\
\hline$B_{6}$ & 1 & 0 & 0 & 0 & 0 & 0 & 0 & 0 & 0 & 0 & 0 & 0 & 0 & 0 & $I$ & 0 & 0 & 0 & \\
\hline$B_{7}$ & 0 & 0 & 1 & 0 & 0 & 0 & 0 & 0 & 1 & 0 & 0 & 1 & 0 & 0 & $I$ & 0 & 0 & 0 & 0 \\
\hline$B_{8}$ & 0 & 1 & 0 & 0 & 0 & 0 & 0 & 0 & 0 & 1 & 0 & 0 & 0 & 0 & 0 & 0 & 0 & 0 & \\
\hline$B_{9}$ & 0 & 0 & 0 & 0 & 0 & 0 & 0 & 0 & 0 & 0 & 0 & 0 & 0 & 1 & 0 & 0 & $I$ & 0 & \\
\hline$B_{10}$ & 0 & 0 & 0 & 0 & 0 & 0 & 0 & 0 & 0 & 0 & 0 & 0 & 0 & 0 & 0 & $I$ & 0 & 0 & 0 \\
\hline $3_{11}$ & 0 & 1 & 0 & 0 & 0 & 0 & 1 & 0 & 0 & 0 & 0 & 0 & 1 & 0 & 0 & 0 & 0 & 0 & 0 \\
\hline $3_{12}$ & 1 & 0 & 0 & 0 & 0 & 0 & 0 & 0 & 0 & 0 & 0 & 0 & 0 & 0 & 0 & 0 & 0 & 0 & $I$ \\
\hline$\beta_{13}$ & 0 & 0 & 0 & 0 & 0 & 1 & 0 & 0 & 0 & 0 & 0 & 0 & 0 & 0 & 0 & 1 & 0 & 0 & \\
\hline & 0 & 1 & 0 & 0 & 0 & 0 & $I$ & 0 & 0 & 1 & 0 & 0 & 0 & 0 & 0 & 0 & 0 & 0 & 0 \\
\hline $3_{15}$ & 0 & 0 & 0 & 0 & 0 & 0 & 0 & 0 & 0 & 0 & 0 & 0 & 0 & 0 & 0 & 1 & 0 & 0 & 0 \\
\hline 3 & 1 & 0 & 0 & 0 & 0 & 0 & 0 & 0 & 0 & 0 & 0 & 0 & 0 & 0 & 0 & 0 & 0 & 0 & 0 \\
\hline & 0 & 0 & 1 & 0 & 0 & 0 & 0 & 0 & 0 & 0 & 0 & 0 & 0 & 0 & 0 & 0 & 0 & 0 & 0 \\
\hline & 0 & 0 & 0 & 0 & 0 & 0 & 0 & 0 & 0 & 0 & 0 & 0 & 0 & 1 & 0 & 0 & 0 & 0 & 0 \\
\hline & 0 & 1 & 0 & 0 & 0 & 0 & 0 & 0 & 0 & 0 & 0 & 0 & 1 & 0 & 0 & 0 & 0 & 0 & 0 \\
\hline & 0 & 0 & 0 & 0 & 0 & 0 & 0 & 0 & 0 & 0 & 1 & 0 & 0 & 0 & 0 & 0 & 0 & 0 & \\
\hline
\end{tabular}

Suppose we consider the ON state of the attribute Good health and nutrition and all other states are OFF the effect of $\mathbf{X}=$ (100000000000000000) on the CDBFCM is given by

$\mathrm{XT}=(010001000001000 \mathrm{I} 000 \mathrm{I})=\mathrm{X}_{1}($ Say $)$

$\mathrm{X}_{1} \mathrm{~T}=(010001000001000 \mathrm{I} 000 \mathrm{I})=\mathrm{X}_{2}($ Say $)=\mathrm{X}_{1}$

$\mathrm{X}_{1}$ is a fixed point of the dynamical system.

Let $\mathbf{Y}=(\mathbf{1 0 1 0 0 0 0 0 0 0 0 0 0 0 0 0 0 0 0 0 0 0 0})$ state vector depicting Good health and nutrition and regular attendance for learning is ON state, dynamical system $B$ is given by

$\mathrm{YT}=(000000010 \mathrm{I} 000000100)=\mathrm{Y}_{1}($ Say $)$

$\mathrm{Y}_{1} \mathrm{~T}=(10 \mathrm{I} 0000 \mathrm{I} 10 \mathrm{I} 00 \mathrm{I} 100100)=\mathrm{Y}_{2}($ Say $)$

$\mathrm{Y}_{2} \mathrm{~T}=(10 \mathrm{I} 0000 \mathrm{I} 10 \mathrm{I} 00 \mathrm{I} 100100)=\mathrm{Y}_{3}($ Say $)=\mathrm{Y}_{2}$
Here $Y_{2}$ is a fixed point of the dynamical system.

\section{Conclusion}

The paper demonstrates by this analysis that programmes must encompass a broader definition involving learners, content, processes, environments and outcomes.

Definitions of quality must be open to change and evolution based on information, changing contexts, and new understandings of the nature of education's challenges. New research ranging from multinational research to action research at the classroom level - contributes to this redefinition. Continuous assessment and improvement can focus on any or all dimensions of system quality: learners, learning environments, content, process and outcomes. 


\section{References}

[1]Adams, D. (1993). Defining educational quality. Improving Educational Quality Project Publication \#1: Biennial Report. Arlington, VA: Institute for International Research.

[2] George J. Klir/ Bo Yuan, "Fuzzy sets and Fuzzy Logic: Theory and Applications", Prentice Hall of India.

[3]W. B. Vasantha Kandasamy and Smarandache Florentin, -Analysis of social aspects of migrant labours living with HIV/AIDS using Fuzzy Theory and Neutrosophic Cognitive Mapsl, Xi-quan, Phoenix (2004).

[4] W. B. Vasantha Kandasamy and Smarandache Florentin, -Fuzzy Cognitive Maps and Neutrosophic Cognitive Mapsll, Xi-quan, Phoenix (2003).

[5] W. B. Vasantha Kandasamy and A. Victor Devadoss, - Some New Fuzzy Techniquesll, Jour. Of Inst.. of. Math \& Comp. Sci. (Math.Ser.), Vol. 17, No.2, (2004), 157-160.

[6] A.Victor Devadoss, M.Clement Joe Anand, "A Solution to Control Suicide in the Domestic Violence using Combined Disjoint Block Fuzzy Cognitive Maps (CDBFCM)", International Journal of Scientific \& Engineering Research, Volume 3, Issue 6, June-2012.

[7]B. Kosko, "Fuzzy Cognitive Maps", International Journal of man-machine studies, January, (1988), 62-75.

[8]H. J. Zimmermann, "Fuzzy Set Theory and its application”, Fourth Edition Springer 2011.
[9]A.Victor Devadoss, M.Clement Joe Anand, A. Felix, "A Study on the Impact of Violent Video-Games playing among Children in Chennai using Neutrosophic Cognitive Maps (NCMs)", International Journal of Scientific \& Engineering Research, Volume 3, Issue 8, August-2012. (article in Press).

[10]A.Victor Devadoss, M.Clement Joe Anand, "Dimensions of Personality of Women in Chennai Using CETD Matrix", International Journal of Computer Applications, July-2012. (article in Press).

[11]B. Kosko, "Hidden patterns in combined and Adaptive Knowledge Networks", Proc. Of the First, IEE International Conference on Neural Networks (ICNN-86(1988) 377-393).

[12]B. Kosko, "Neural Networks and Fuzzy systems: A Dynamical System Approach to Machine Intelligence", Prentice Hall of India, 1997.

[13]George J.Klir/Bo Yuan, "Fuzzy sets and Fuzzy Logic: Theory and Applications", Prentice Hall of India.

[14]Programme evaluatin report: Activity Based Learning Tamil Nadu, National council of educational research and training, December 2011.

[15]R. Axelrod, "Structure of decision: The cognitive maps of political elites". Princeton, N.J: Princeton University Press, 1976.

[16]H. J. Zimmermann, -Fuzzy Set Theory and its application\|, Fourth Edition Springer 2011. 